\author{
Oleh Matskiv ${ }^{1}$, Agnieszka Kusiak ${ }^{2,3}$, Beata Zwierko ${ }^{3}$, Marta Dura ${ }^{3,4}$, Jacek Budzyński ${ }^{1}$ \\ ${ }^{1}$ Department of Vascular and Internal Diseases, Nicolaus Copernicus University in Torun Ludwik Rydygier Collegium Medicum \\ in Bydgoszcz, Poland \\ ${ }^{2}$ Doctoral School of Medical and Health Sciences, Nicolaus Copernicus University in Torun Ludwik Rydygier Collegium Medicum \\ in Bydgoszcz, Poland \\ ${ }^{3}$ Department of Radiology and Diagnostic Imaging, Jan Biziel University Hospital No. 2 in Bydgoszcz, Poland \\ ${ }^{4}$ University Chair of Diagnostic Imaging, Nicolaus Copernicus University in Torun, Ludwik Rydygier Collegium Medicum in Bydgoszcz, Poland
}

\title{
The risk factors for all-cause in-hospital death in patients with acute pulmonary embolism
}

\section{Corresponding author:}

Jacek Budzyński, Department of Angiology, Jan Biziel University Hospital No. 2 in Bydgoszcz, 75 Ujejskiego Str., 85-168 Bydgoszcz, Poland; e-mail: jb112233@cm.umk.pl

Medical Research Journal 2021; Volume 6, Number 4, 322-329 10.5603/MRJ.2021.0058 Copyright (C) 2021 Via Medica ISSN 2451-2591 e-ISSN 2451-4101

\begin{abstract}
Introduction: Patient risk stratification is important in managing individuals with suspected acute pulmonary embolism (APE). The aim of this study was to determine risk factors for in-hospital mortality among real-world patients who had undergone computed tomography pulmonary angiography (CTPA) due to suspected APE.

Material and methods: Retrospective analysis of clinical data extracted from the medical documentation of 700 consecutive patients in whom CTPA was performed due to APE suspicion.

Results: APE was confirmed in $22.7 \%$ of the patients in the sample. In-hospital death was recorded in $10.1 \%$ and $12.4 \%$ of patients with and without APE confirmed in CTPA, respectively. APE-related death was diagnosed in $37.5 \%$ of the APE patients who died during hospitalization. Compared to patients who were discharged from hospital, those who died during hospitalization had a greater prevalence of comorbidities (e.g., neoplasm) and higher values of laboratory determinations and prognostic rule scores. An age-adjusted high-sensitivity troponin I (hs-TNI) cut-off and Pulmonary Embolism Severity Index (PESI) score were found to be independent risk factors of in-hospital death, but only in the whole study group and in patients without APE confirmed in CTPA. The area-under-the-curve value for all the parameters studied was lower than 0.6 .

Conclusions: Age-adjusted hs-TNI cut-off and PESI score were independent risk factors for in-hospital death in patients with APE suspicion. The predictive power of standard stratifying tools is insufficient in real-world patients with suspected APE. Patients with suspected APE require careful diagnosis and management of comorbidities because these may affect the in-hospital mortality rate.

Key words: computed tomography pulmonary angiography, in-hospital mortality, pulmonary embolism, retrospective study, risk assessment
\end{abstract}

Med Res J 2021; 6 (4): 322-329

\section{Introduction}

The aggressiveness of acute pulmonary embolism (APE) management is related to the severity of the clinical course and a prognosis of early mortality. In order to widen the range of therapeutic strategies, reliable tools for risk stratification are required to identify qualifying patients. A strategy for APE patient management, especially for normotensive patients, consists of: (a) estimating the risk of deep vein thrombosis (DVT), e.g., using the Padua or Caprini rule, and DVT diagnosis; (b) assessment of the risk of APE on the basis of empirical clinical judgment, Wells or Geneva score, or D-dimer cut-offs (standard and age- adjusted); (c) making an APE diagnosis on the basis of symptoms and imaging in computed tomography pulmonary angiography (CTPA), including identifying the type of embolus (e.g., air, fluid, fat, or solid materials, such as thrombus or carcinomatous cells) and making a differential diagnosis in order to exclude other diseases of the pulmonary arteries or other causes of the symptoms presented; (d) classifying APE severity and determin- 
ing early (in-hospital or 30-day) mortality probability; (e) decisions concerning potential indications and contraindications for the various kinds of APE therapy being considered on the basis of the outcomes of the patient's work-up (e.g., anticoagulants as home or in-hospital [with or without early discharge] therapy, systemic or catheter-directed thrombolysis, surgical embolectomy, or percutaneous thrombectomy) [1]; (f) the application of APE treatment; and (g) assessing the type and strength of provoking factors for a thromboembolic event (e.g., a transient provoking risk factor [strong, moderate, mild], unprovoked idiopathic APE, coexisting neoplasm [cancer-associated thrombosis (CAT)], or pregnancy) that could affect the risk of DVT recurrence, in order to guide the duration of anticoagulation in secondary and prolonged thromboembolism prevention [2-4]. One important part of the aforementioned strategy is the assessment of APE severity and APE-related risk with respect to the probability of early (in-hospital or 30-day) death; this type of assessment, as stated above, influences not only the patient's prognosis but also the aggressiveness of the therapy undertaken (risk-adjusted management). The recent 2019 European Society of Cardiology (ESC) recommendations on APE management [3] suggest APE severity assessment on the basis of the presence of hypotension (high-risk patients) and, in normotensive patients, the use of stratification tools. Such tools include: (a) calculation of Pulmonary Embolism Severity Index (PESI) or simplified PESI (sPESI) scores and the patient's qualification to one of five PESI risk classes or to one of two SPESI classes; (b) ESC classification of pulmonary embolism severity and the risk of early death (low/intermediate-low/intermediate-high/high); (c) determination of blood high-sensitivity troponin I (hs-TNI) or high-sensitivity troponin T (hs-TNT) concentration cut-offs (standard and age-adjusted); (d) assessment for signs of right ventricular (RV) pressure overload in transthoracic echocardiography (TTE) or RV enlargement in CTPA; and, in some cases, (e) determination of blood $\mathrm{N}$-terminal proBtype natriuretic peptide (NT-proBNP) concentration cut-off (mostly $\geq 600 \mathrm{pg} / \mathrm{ml}$ ) [2, 3]. Researchers have also suggested using the following stratifying tools: the presence of coexisting DVT in patients at intermediate-low risk of short-term complications [5]; (b) selected parameters of CTPA imaging [6-8], as well as various combinations of the parameters, some of which are mentioned above, such as (i) D-dimer cut-off with sPESI [9], (ii) PESI with NT-proBNP, hs-TNT, and high-sensitivity C-reactive protein cut-offs [10] and (iii) PESI, sPESI and Bova scores with additional patient stratification on the basis of systolic blood pressure [11]; and (c) a combination of a quick Sequential Organ Failure Assessment (qSOFA) score with particular ECG signs [12].
As real-world data on the impact of advances in APE patient risk stratification on in-hospital mortality are limited, we performed a retrospective analysis in order to determine the prognostic power of standard stratifying tools in the prediction of in-hospital death among consecutive, real-world patients with suspected APE who underwent CTPA during three years in one Polish university hospital.

\section{Material and methods}

\section{Patients}

We conducted a retrospective analysis of the medical documentation of 700 consecutive real-world inpatients who underwent CTPA due to suspected APE between January 2017 and January 2020. Patients were selected on the basis of a chest angiography referral, regardless of the ward to which they had been admitted.

\section{Methods}

For all consecutive patients with suspected APE, we extracted the following from the electronic medical database: demographic data, presenting symptoms, and descriptions of CTPA performed by an experienced radiologist. The occurrence of in-hospital all-cause death and details of the causes of those deaths were also analyzed.

We calculated the following on the basis of the available data: (a) Padua and Caprini prediction scores for DVT risk assessment; (b) original (with three levels: low/intermediate/high) and simplified (with two levels: unlikely, likely) Wells and Geneva scores for DVT probability assessment; (c) standard ( $\geq 500 \mathrm{ng} / \mathrm{ml}$ ) and age-adjusted (age $\times 10 \mathrm{ng} / \mathrm{mL}$, for patients aged $>50 \mathrm{y}$ ) cut-offs for blood D-dimer concentrations [2, 3]; (d) original and simplified Wells and Geneva scores for APE risk assessment; (e) PESI and sPESI scores; (f) prevalence of an intermediate risk of in-hospital death according to 2014 ESC Guidelines; (g) prevalence of the respective classes of pulmonary embolism severity and the risk of early (in-hospital or 30-day) death on the basis of hypotension or the presence of shock, and/or signs of RV pressure overload in TTE or RV enlargement in CTPA; blood hs-TNI concentration above established cut-offs (standard: $0.014 \mathrm{ng} / \mathrm{ml}$; age-adjusted: age < 75y, hs$\mathrm{TNI} \geq 0.014 \mathrm{ng} / \mathrm{ml}$; age $\geq 75 \mathrm{y}$, hs-TNI $\geq 0.045 \mathrm{ng} / \mathrm{ml}$ [3]), and a blood NT-proBNP concentration cut-off $\geq 600 \mathrm{pg} / \mathrm{ml}$ according to 2019 ESC Guidelines [3].

\section{Outcomes measured}

The primary outcome measured was all-cause in-hospital death. 


\section{Bioethics}

The study protocol was approved by a local Bioethical Committee at Ludwik Rydygier Collegium Medicum in Bydgoszcz, Nicolaus Copernicus University No. $161 / 2020$, on March 31,2020 . The analyses were conducted in compliance with the Declaration of Helsinki for medical research. This study was registered in our hospital under No. 33/2020.

\section{Statistics}

Statistical analysis was conducted using the licensed version of the statistical software Statistica version 13.1 (data analysis software) developed by Tibco Software, Inc. (2017). The statistical significance level was set at a $p$-value of $<0.05$. The normal distribution of the study variables was verified using the Kolmogorov-Smirnov test. The power of all statistically significant comparisons was at least $90 \%$. The results were presented as the mean \pm standard deviation, or $n, \%$. The statistical significance of differences between groups was verified using the Student's t-test for parametric quantitative variables and the $\mathrm{Chi}^{2}$ test for qualitative variables. Logistic regression was used to determine the odds ratios (OR) and 95\% confidence intervals (95\% $\mathrm{Cl}$ ) of independent factors associated with in-hospital death for the whole study group, for patients with APE, and for patients without APE confirmed in CTPA. The discrimination of each risk score in predicting in-hospital mortality was also calculated by measuring the area under the curve (AUC) with a $95 \% \mathrm{Cl}$ in receiver operating characteristic (ROC) analysis.

\section{Results}

An APE diagnosis was confirmed in 159 (22.7\%) of all 700 patients who underwent CTPA due to suspected APE during the three-year period examined in our study (Tab. 1). In terms of the whole study group, in-hospital mortality amounted to $11.9 \%$, affecting $10.1 \%$ and $12.4 \%$ of patients with and without APE confirmed in CTPA ( $p=0.34)$, respectively. When patients were grouped in relation to the five (I-V) PESI classes, in-hospital mortality amounted to $2.7 \%, 5.6 \%, 7.9 \%, 13.8 \%$, and $31.4 \%$, respectively. Whereas, among high-risk patients classified with regard to an SPESI score $\geq 1,15.3 \%$ of patients died during hospitalization. We also found that, in relation to ESC classification by APE severity and early mortality [3], for patients with low, intermediate-low, intermediate-high, or high risk of early mortality, the in-hospital death rates amounted to $4.4 \%, 10.7 \%$, $16.5 \%$, and $34.1 \%$, respectively. Among patients with APE confirmed in CTPA, the most prevalent diagnoses of the cause of death were APE-related (37.5\%), pneumonia (37.5\%), stroke (12.5\%), and sepsis (12.0\%). However, among patients without confirmation of APE, the most prevalent final diagnoses of thecause of death were: pneumonia (28.4\%), sepsis $(22.4 \%)$, cardiac disorders $(17.9 \%)$, end-stage cancerous disease (11.9\%), and stroke (10.5\%).

Compared to patients who were discharged, patients with APE confirmed in CTPA who died during hospitalization had a significantly higher prevalence of cancerous disease, higher Padua and Caprini scores for DVT risk assessment, and higher blood D-dimer concentration, with no difference in prevalence regarding the established cut-offs; higher blood hs-TNI concentration and were more likely to be above the age-adjusted cut-off; higher PESI and SPESI scores, higher prevalence among those in PESI risk class $V$ and those with an SPESI risk class $\geq 1$; as well as being less likely to be in a low-risk category and more likely to be in the intermediate-high and high classes of ESC classification of APE severity and risk of early mortality (Tab. 1). Similar differences between patients who died and those who survived were observed among individuals without APE confirmed in CTPA when we compared the respective subgroups of patients with and without APE confirmation in CTPA (Tab. 1). Among individuals who died during hospitalization, those with APE confirmed in CTPA had a greater prevalence of a high category of risk of early death according to ESC classification than patients without an APE confirmation in CTPA (Tab. 1).

\section{Risk factors for in-hospital death}

Using univariate analysis, we found that patients with higher scores for the prognostic rules and those who were above the biochemical parameter cut-off thresholds were more likely to die during hospitalization than their counterparts (data not presented). However, in multivariate analysis, performed using a multiple logistic regression method, the independent risk factors for in-hospital all-cause death for the whole study group and in patients without an APE confirmation in CTPA were age-adjusted hs-TNI cut-off and the class of risk according to PESI score (Tab. 2). Among patients with a confirmation of APE in CTPA, none of the known risk factors for early mortality was statistically significant (Tab. 2).

\section{The diagnostic power of the parameters studied to predict in-hospital all-cause mortality}

We also performed ROC analysis to determine the AUC for the respective stratifying parameters (Table 3 ). Almost all the parameters analyzed were statistically significant in predicting the occurrence of in-hospital death, although we failed to determine statistically 
Table 1. Clinical characteristics of patients with pulmonary embolism confirmed in CTPA in relation to in-hospital death

\begin{tabular}{|c|c|c|c|c|}
\hline \multirow[t]{2}{*}{ Parameter } & \multicolumn{2}{|c|}{$\begin{array}{l}\text { With APE confirmed } \\
\text { in CTPA }(n=159)\end{array}$} & \multicolumn{2}{|c|}{$\begin{array}{l}\text { Without APE confirmed } \\
\text { in CTPA }(n=541)\end{array}$} \\
\hline & $\begin{array}{c}\begin{array}{c}\text { In-hospital } \\
\text { death }\end{array} \\
\text { (n = 16; 10.1\%) }\end{array}$ & $\begin{array}{c}\text { Discharged } \\
\text { from hospital } \\
(n=143 ; 89.9 \%)\end{array}$ & $\begin{array}{c}\text { In-hospital } \\
\text { death } \\
\text { (n = 67; 12.4) }\end{array}$ & $\begin{array}{c}\text { Discharged } \\
\text { from hospital } \\
(n=474 ; 87.6 \%)\end{array}$ \\
\hline Male gender (n, \%) & $9(56.3 \%)$ & $64(44.8 \%)$ & $32(47.8 \%)$ & $216(45.6 \%)$ \\
\hline Age (years) & $69.13 \pm 6.50$ & $65.11 \pm 20.52$ & $70.8 \pm 14.7$ & $65.16 \pm 16.2^{*}$ \\
\hline History of cancerous disease $(n, \%)$ & $6(37.5 \%)$ & $28(19.6 \%)^{*}$ & $11(16.4 \%) \#$ & $81(17.1 \%)$ \\
\hline DVT diagnosis $(n, \%)$ & $2(12.5 \%)$ & $45(31.5 \%)$ & $6(9.0 \%)$ & $51(10.8 \%)$ \\
\hline Bilateral pulmonary embolism (n, \%) & $9(56.3 \%)$ & $101(70.6 \%)$ & 0 & 0 \\
\hline Pulmonary artery dilatation (n, \%) & $8(50.0 \%)$ & $93(65.0 \%)$ & $22(32.8 \%)$ & $180(38.0 \%)$ \\
\hline $\begin{array}{l}\text { Padua Prediction Score for DVT risk } \\
\text { assessment }\end{array}$ & $4.78 \pm 3.23$ & $2.71 \pm 2.64^{*}$ & $3.7 \pm 2.8 \#$ & $2.3 \pm 2.1^{*}$ \\
\hline Padua Prediction Score $\geq 4$ (n, \%) & $5(31.3 \%)$ & $35(24.5 \%)$ & 27 (40.3\%) & $91(19.2 \%)^{*}$ \\
\hline Caprini score for DVT risk assessment & $5.64 \pm 2.54$ & $2.63 \pm 1.72^{*}$ & $3.4 \pm 2.1 \#$ & $2.96 \pm 2.1$ \\
\hline Caprini score for DVT risk (2/3) (n, \%) & $\begin{array}{l}4(25.0 \%) / \\
6(37.5 \%)\end{array}$ & $\begin{array}{l}43(30.1 \%) / \\
9(6.3 \%)^{*}\end{array}$ & $\begin{array}{l}22(32.8 \%) / \\
13(19.4 \%)\end{array}$ & $\begin{array}{l}96(20.3 \%) / \\
40(8.4 \%)\end{array}$ \\
\hline Original Wells score & $2.31 \pm 1.66$ & $3.03 \pm 2.37$ & $2.2 \pm 1.8$ & $1.6 \pm 1.7^{*}$ \\
\hline Simplified Wells score & $1.63 \pm 1.15$ & $1.60 \pm 1.10$ & $1.35 \pm 1.0$ & $0.95 \pm 0.9^{\star} \#$ \\
\hline $\begin{array}{l}\text { Clinical probability of APE according to } \\
\text { simplified Wells score (likely) (n, \%) }\end{array}$ & $7(43.8 \%)$ & $73(51.0 \%)$ & $26(38.8 \%)$ & $109(23.0 \%)^{\star} \#$ \\
\hline Geneva score & $6.06 \pm 3.55$ & $6.68 \pm 2.88$ & $6.0 \pm 2.5$ & $5.0 \pm 2.6^{*}$ \\
\hline $\begin{array}{l}\text { Clinical probability of APE according to } \\
\text { simplified Geneva score (likely) (n, \%) }\end{array}$ & $12(75.0 \%)$ & $88(61.5 \%)$ & 38 (56.7\%)\# & $192(40.5 \%)^{\star} \#$ \\
\hline $\begin{array}{l}\text { D-dimer (ng/ml) } \\
\text { (data available for } 509 \text { patients) }\end{array}$ & $20585 \pm 19550$ & $10268 \pm 10092^{*}$ & $\begin{array}{l}12667.1 \pm \\
14397.5\end{array}$ & $4299.43 \pm 7613.7^{*}$ \\
\hline $\begin{array}{l}\text { Age-adjusted D-dimer cut-offs (10*age) } \\
(\mathrm{n}, \%)\end{array}$ & $12(100 \%)$ & $100(97.0 \%)$ & 52 (100\%) & 308 (90.3\%) \\
\hline $\begin{array}{l}\text { Signs of RV overload in transthoracic } \\
\text { echocardiography (data available for } 128 \\
\text { patients) (n, \%) }\end{array}$ & $2(12.5 \%)$ & $52(36.4 \%)$ & $7(10.4 \%)$ & $67(14.1 \%)$ \\
\hline $\begin{array}{l}\text { hs-TNI (ng/ml) } \\
\text { (data available for } 544 \text { patients) }\end{array}$ & $0.28 \pm 0.52$ & $0.09 \pm 0.22^{*}$ & $0.2 \pm 0.3$ & $0.1 \pm 0.2^{*}$ \\
\hline hs-TNI $\geq 0.014$ ng/ml (n, \%) & $14(100 \%)$ & $93(79.5 \%)$ & $51(94.4 \%)$ & $249(68.8 \%)^{\star} \#$ \\
\hline $\begin{array}{l}\text { Age-adjusted troponin cut-offs } \\
\text { (age < 75y, troponin } \geq 0.014 \mathrm{ng} / \mathrm{ml} ; \text { age } \geq \\
75 \mathrm{y}, \text { troponin } \geq 0.045 \mathrm{ng} / \mathrm{ml})(\mathrm{n}, \%)\end{array}$ & $13(92.9 \%)$ & $77(65.8 \%)$ * & $49(90.7 \%)$ & $185(51.5 \%)^{\star} \#$ \\
\hline $\begin{array}{l}\text { NT-proBNP (pg/ml) } \\
\text { (data available for } 401 \text { patients) }\end{array}$ & $4233.75 \pm 4115.9$ & $4069.34 \pm 6584.4$ & $\begin{array}{l}8504.2 \pm \\
10019.2 \#\end{array}$ & $4245.42 \pm 80818.2^{\star} \#$ \\
\hline NT-proBNP $\geq 600$ pg/ml (n, \%) & $7(87.5 \%)$ & $79(67.0 \%)^{*}$ & 25 (92.6\%) & $150(60.5 \%)^{*}$ \\
\hline PESI score & $142.94 \pm 44.4$ & $93.85 \pm 34.95^{\star}$ & $124.83 \pm 38.5$ & $92.03 \pm 33.4^{*}$ \\
\hline $\begin{array}{l}\text { Risk class according to PESI score } \\
(\mathrm{III} / \mathrm{IV} / \mathrm{V})(\mathrm{n}, \%)\end{array}$ & $\begin{array}{l}4(25.0 \%) / \\
2(12.5 \%) / \\
10(62.5 \%)\end{array}$ & $\begin{array}{l}40(28.0 \%) / \\
16(11.2 \%) / \\
27(18.9 \%)^{*}\end{array}$ & $\begin{array}{c}10(14.9) / \\
10(14.9 \%) / \\
34(50.7 \%)\end{array}$ & $\begin{array}{l}124(26.2 \%) / \\
59(12.4 \%) / \\
69(14.6 \%)^{*}\end{array}$ \\
\hline sPESI score & $2.31 \pm 1.40$ & $1.24 \pm 1.17^{*}$ & $2.2 \pm 1.3$ & $1.4 \pm 4.8$ \\
\hline $\begin{array}{l}\text { Risk class according to sPESI score } \\
(\geq 1 \text { score) }(n, \%)\end{array}$ & $16(100 \%)$ & $96(67.1 \%)^{\star}$ & $57(85.1 \%)$ & $308(65.0 \%)^{*}$ \\
\hline $\begin{array}{l}\text { ESC classification of APE severity and } \\
\text { risk of early death: low/ intermediate-low/ } \\
\text { intermediate-high/high (n, \%) }\end{array}$ & $\begin{array}{c}0(0 \%) / \\
1(6.3 \%) / \\
6(37.5 \%) / \\
9(56.3 \%)\end{array}$ & $\begin{array}{c}58(40.6 \%) / \\
10(7.0 \%) / \\
48(33.6 \%) / \\
27(18.9 \%)^{\star}\end{array}$ & $\begin{array}{c}12(17.9 \%) / \\
11(16.4 \%) / \\
39(58.2 \%) \\
5(7.5 \%)\end{array}$ & $\begin{array}{c}204(43.0 \%) / \\
90(19.0 \%) / \\
180(38.0 \%)^{\star} \# \\
0\end{array}$ \\
\hline
\end{tabular}

APE — acute pulmonary embolism; CTPA — computed tomography pulmonary angiography; DVT — deep vein thrombosis; ESC — European Society of Cardiology; hs-TNI — high-sensitivity troponin I; NT-proBNP — N-terminal proB-type natriuretic peptide; PESI — Pulmonary Embolism Severity Index; RV — right ventricular; SPESI - simplified Pulmonary Embolism Severity Index. * $-p<0.05$ for differences between values obtained between subgroups among patients with and without APE confirmation; \#-p $<0.05$ for differences between subgroups of patients with and without APE confirmation 
Table 2. Risk factors for in-hospital death for the whole study group and in patients with and without APE confirmed in CTPA: multiple logistic regression results

\begin{tabular}{|c|c|c|c|c|c|c|}
\hline \multirow[t]{2}{*}{ Parameter } & \multicolumn{2}{|c|}{ Whole study group } & \multicolumn{2}{|c|}{$\begin{array}{l}\text { Patients with APE } \\
\text { confirmed in CTPA }\end{array}$} & \multicolumn{2}{|c|}{$\begin{array}{l}\text { Patients without APE } \\
\text { confirmed in CTPA }\end{array}$} \\
\hline & OR; $95 \% \mathrm{Cl}$ & $\mathbf{p}$ & OR; $95 \% \mathrm{Cl}$ & $\mathbf{p}$ & OR; $95 \% \mathrm{Cl}$ & $\mathbf{p}$ \\
\hline Age-adjusted blood hsTNI concentration & $5.9 ; 2.6-13.4$ & $<0.01$ & $2.2 ; 0.2-20.8$ & 0.48 & $7.1 ; 2.8-17.7$ & $<0.01$ \\
\hline NT-proBNP $\geq 600$ pg/ml & $1.2 ; 0.5-2.8$ & 0.64 & $1.8 ; 0.2-17.4$ & 0.60 & $1.6 ; 0.6-4.6$ & 0.30 \\
\hline PESI (likely) & $2.6 ; 1.6-4.3$ & $<0.01$ & $0.9 ; 0.1-12.1$ & 0.90 & $2.2 ; 1.6-3.1$ & $<0.01$ \\
\hline $\begin{array}{l}\text { ESC classification of pulmonary } \\
\text { embolism severity and risk of early death }\end{array}$ & $0.6 ; 0.2-1.4$ & 0.21 & $6.2 ; 0.1-62.4$ & 0.43 & $0.6 ; 0.4-1.1$ & 0.16 \\
\hline
\end{tabular}

$\mathrm{APE}$ - acute pulmonary embolism; $\mathrm{Cl}$ - confidence interval; CTPA = computed tomography pulmonary angiography/angiogram; ESC — European Society of Cardiology; hs-TNI — high-sensitivity troponin I; NT-proBNP — N-terminal proB-type natriuretic peptide; OR — odds ratio; PESI — Pulmonary Embolism Severity Index

Table 3. AUC in ROC analysis of the power to predict risk of in-hospital mortality for the whole study group and in patients with and without APE confirmed in CTPA

\begin{tabular}{|c|c|c|c|}
\hline \multirow[t]{2}{*}{ Parameter } & Whole study group & APE confirmed in CTPA & APE not confirmed in CTPA \\
\hline & AUC; $95 \% \mathrm{Cl} ; \mathrm{p}$ & AUC; $95 \% \mathrm{Cl} ; \mathrm{p}$ & AUC; $95 \% \mathrm{Cl} ; \mathrm{p}$ \\
\hline Age & $0.45 ; 0.35-0.48 ; p<0.01$ & $0.48 ; 0.37-0.59 ; p=0.71$ & $0.40 ; 0.33-0.47 ; p<0.01$ \\
\hline Male gender & $0.48 ; 0.41-0.55 ; p=0.55$ & $0.48 ; 0.30-0.60 ; p=0.49$ & $0.49 ; 0.41-0.56 ; p=0.73$ \\
\hline Padua score & $0.36 ; 0.28-0.44 ; p<0.01$ & $0.31 ; 0.12-0.50 ; p=0.05$ & $0.36 ; 0.27-0.46 ; p<0.01$ \\
\hline Caprini score & $0.39 ; 0.32-0.47 ; p<0.01$ & $0.15 ; 0.04-0.27 ; p<0.01$ & $0.45 ; 0.37-0.53 ; p=0.19$ \\
\hline Original Wells score & $0.43 ; 0.37-0.49 ; p=0.02$ & $0.58 ; 0.47-0.70 ; p=0.16$ & $0.38 ; 0.31-0.45 ; p<0.01$ \\
\hline Simplified Wells score & $0.41 ; 0.35-0.47 ; p<0.01$ & $0.51 ; 0.38-0.65 ; p=0.84$ & $0.38 ; 0.31-0.45 ; p<0.01$ \\
\hline $\begin{array}{l}\text { Three-level original Wells APE } \\
\text { probability }\end{array}$ & $0.40 ; 0.34-0.46 ; p<0.01$ & $0.47 ; 0.33-0.61 ; p=0.66$ & $0.40 ; 0.34-0.46 ; p<0.01$ \\
\hline Original Geneva score & $0.44 ; 0.37-0.50 ; p=0.04$ & $0.55 ; 0.40-0.70 ; p=0.49$ & $0.39 ; 0.33-0.46 ; p=0.043$ \\
\hline $\begin{array}{l}\text { Three-level original Geneva APE } \\
\text { probability }\end{array}$ & $0.44 ; 0.38-0.50 ; p=0.06$ & $0.54 ; 0.38-0.69 ; p=0.66$ & $0.41 ; 0.34-0.48 ; p<0.01$ \\
\hline Simplified Geneva score (likely) & $0.42 ; 0.35-0.48 ; p=0.01$ & $0.46 ; 0.31-0.61 ; p=0.56$ & $0.42 ; 0.35-0.48 ; p=0.012$ \\
\hline Blood D-dimer concentration & $0.25 ; 0.19-0.30 ;<0.01$ & $0.36 ; 0.17-0.55 ; p=0.15$ & $0.19 ; 0.14-0.25 ; p<0.01$ \\
\hline Blood hs-TNI concentration & $0.23 ; 0.18-0.29 ;<0.01$ & $0.29 ; 0.16-0.43 ; p<0.01$ & $0.21 ; 0.15-0.27 ; p<0.01$ \\
\hline Blood NT-proBNP concentration & $0.30 ; 0.23-0.38 ;<0.01$ & $0.38 ; 0.22-0.53 ; p=0.12$ & $0.27 ; 0.19-0.36 ; p<0.01$ \\
\hline $\begin{array}{l}\text { Intermediate APE severity } \\
\text { according to } 2014 \text { ESC } \\
\text { Guidelines }\end{array}$ & $0.38 ; 0.32-0.44 ;<0.01$ & $0.41 ; 0.27-0.54 ; p=0.17$ & $0.38 ; 0.32-0.44 ; p<0.01$ \\
\hline PESI score & $0.25 ; 0.19-0.30 ;<0.01$ & $0.19 ; 0.09-0.28 ; p<0.01$ & $0.26 ; 0.19-0.32 ; p<0.01$ \\
\hline $\begin{array}{l}\text { PESI class of early mortality risk } \\
(I-V)\end{array}$ & $0.26 ; 0.20-0.31 ;<0.01$ & $0.20 ; 0.11-0.30 ; p<0.01$ & $0.27 ; 0.20-0.34 ; p<0.01$ \\
\hline sPESI score & $0.28 ; 0.22-0.34 ;<0.01$ & $0.28 ; 0.16-0.39 ; p<0.01$ & $0.28 ; 0.12-0.35 ; p<0.01$ \\
\hline $\begin{array}{l}\text { Early mortality risk sPESI class } \\
(\geq 1)\end{array}$ & $0.39 ; 0.33-0.45 ; p<0.01$ & $0.33 ; 0.23-0.45 ; p<0.01$ & $0.41 ; 0.34-0.47 ; p<0.01$ \\
\hline $\begin{array}{l}\text { ESC classification of pulmonary } \\
\text { embolism severity and risk of } \\
\text { early death }\end{array}$ & $0.41 ; 0.34-0.47 ; p<0.01$ & $0.31 ; 0.20-0.42 ; p<0.01$ & $0.49 ; 0.40-0.58 ; p=0.82$ \\
\hline
\end{tabular}

APE — acute pulmonary embolism; AUC — area under the curve; $\mathrm{Cl}$ - confidence interval; CTPA — computed tomography pulmonary angiography/angiogram; ESC - European Society of Cardiology; hs-TNI - high-sensitivity troponin I; NT-proBNP — N-terminal proB-type natriuretic peptide; PESI — Pulmonary Embolism Severity Index; ROC — receiver operating characteristic; sPESI — simplified Pulmonary Embolism Severity Index 
and clinically significant cut-offs in the three patient subgroups analyzed (Tab. 3). The greatest AUCs were obtained for patients' age (the whole study group), early mortality risk sPESI class $(\geq 1)$ (in patients without APE confirmed in CTPA), and simplified Geneva score (for patients without APE confirmed in CTPA). However, none of the parameters and/or rules studied reached a statistically significant AUC with values greater than 0.6 , which indicates the low clinical usefulness of the parameters studied in real-world patients suspected of having APE.

\section{Discussion}

Among the large sample of consecutive real-world patients who had undergone CTPA due to suspected APE that we studied, the average overall risk of all-cause in-hospital death was high and amounted to $10-12 \%$. When we compared our patients with data reported in recent ESC Guidelines on APE management, the average mortality rate observed was also higher in PESI classes III-V (30-day mortality ranged from 3.2-7.1\%, $4.0-11.4 \%$, and $10.0-24.5 \%$, respectively) and in high-risk patients stratified according to the sPESI rule (10.9\%) [3]. The risk of all-cause in-hospital death in our study was also higher than in meta-analyses by Barco et al. [13] and Elias et al. [14], who showed early all-cause mortality rates in low-risk APE patients amounted to 1.5-2.3\%, and $10.7-11.4 \%$ in the high-risk group. Other authors also reported lower APE patient mortality than we found in our study. For example a $1.2 \%$ mortality rate among low-risk APE patients classified using PESI and SPESI score [10], a 6.4\% 30-day adverse outcome rate among normotensive (non-high-risk) patients [16], a $7.7-10 \% 30$-day mortality rate in the overall group [17, 18 ], and a $2.1 \%$ and $23.0 \%$ mortality rate for low- and high-risk APE patients [7]. Ebner et al. [19] and Soriano et al. [20] showed similar rates to those observed by us for in-hospital all-cause mortality, which in their studies amounted to $12.2 \%$ for the overall patient cohort [19], $3.25 \%$ for an sPESI score of 0 and $19.51 \%$ for an sPESI score $\geq 1$, and $20 \%$ among those with a high-risk PESI score (classes III-V) [20].

In our study, we found that clinical factors associated with a complicated APE course were also useful in predicting in-hospital all-cause mortality among patients in whom APE was not confirmed in CTPA (Tab. 2). However, multivariate analysis showed only that age-adjusted hs-TNI cut-off and PESI score (likely) were independent factors influencing the risk of in-hospital death. The strength of these relationships in our study, expressed by OR values, was similar to that reported by Darwish et al. [15] for hs-TNI, by Barco et al. [13] for NT-proBNP, and by Soriano et al. [20] for PESI score. However, it should be underlined that in our study group, even among patients with APE confirmed in CTPA, APE was documented as the final cause of death in only $37.5 \%$ of patients. The other main causes of death in the APE group included pneumonia and sepsis, similar to patients in whom suspected APE was not confirmed. These data corroborate reported causes of long-term mortality after massive, submassive, and low-risk pulmonary embolism: malignancy, cardiac disease, respiratory disease, and APE [17].

To determine the predictive power of the parameters studied, we also performed ROC analysis for the whole study group and patients with and without APE confirmed in CTPA (Table 3). However, we failed to determine clinically useful cut-off values for the parameters we studied in order to analyze their predictive power or to create and validate combinations of these parameters. Moreover, the AUC-ROC obtained for the parameters studied was lower than 0.6 , which indicates that they have insufficient power for use in predicting in-hospital mortality among a real-world population of patients who had undergone CTPA due to suspected APE. Higher AUC-ROC values than were obtained in our study (approximately $0.25-0.45$; Tab. 3 ) in the prediction of APE complications were found, for example, by Jia et al. [8] for a combination of RV to left ventricular (RV/LV) volume ratio, central pulmonary artery embolism and right atria/left atria $(R A / L A)$ volume ratio $(A \cup C=0.86)$; by Kozłowska et al. [9] using a stratification model combining SPESI score with a D-dimer cut-off $>1.35 \mathrm{mg} / \mathrm{L}$ (AUC = 0.67); by Vamsidhar et al. [21] using RV/LV volume ratio (AUC $=0.94$, Qanadli index, which is a measure of clot burden in CTPA, and NT-proBNP $(A \cup C=0.81)$; and by Jen et al. [7], who obtained the following AUC for PESI, SPESI, and ESC classification of APE severity and early mortality scores: $0.67,0.58$ and 0.65 , respectively. In a study by Barnes et al. [11], each of the risk stratification tools for APE patients had a modest level of discrimination for 7-day mortality (AUC range: $0.62-0.67$ ), with slightly lower discrimination for 30-day mortality (AUC range: 0.55-0.69). The most likely cause of these inconsistencies between our results and the findings of the other authors is the comorbidity burden on the real-world patients in our study, which was demonstrated, for example, by the diagnoses of death given by the lead doctors in our hospital. In our study, this comorbidity burden was also demonstrated by a greater prevalence of cancerous disease, as well as by higher Padua and Caprini scores (Tab. 1). The other potential cause of the observed discrepancies was the retrospective calculation of DVT and APE probability and severity scores, which in many cases was based on documented values of the parameters analyzed and might not always reflect the true clinical scenario of the patient, and thus could be a source of bias. On the other 
hand, our observations showed that independent of the presence of APE signs in CTPA, every patient needs personalized management and to have comorbidities that may potentially affect his or her prognosis taken into account, as well as indications and contraindications for standard management.

Our study may also suggest underuse of risk assessment among real-world patients with suspected APE. This statement can be supported by our finding that only $22.7 \%$ of the CTPA examinations performed were positive for APE, that blood D-dimer concentration was determined in only 509 (72.7\%) of the patients studied, and transthoracic echocardiography was performed before ordering CTPA in only 128 patients (18.3\%). On the other hand, the percentage of patients with a D-dimer determination performed before CTPA in our study was greater than in the study by Perera et al. [22], who reported that $D$-dimer determination was documented in only $64 / 344$ (18.4\%) of the cases analyzed. Perera et al. [22] suggested that more than $50 \%$ of CTPA examinations performed were unwarranted, due to inadequate pre-test probability estimation. This demonstrates the need to introduce APE and DVT diagnostic algorithms into everyday real-world clinical practice, in order to reduce patients' exposure to X-rays and to increase the efficiency of resource utilization.

\section{Study limitations}

As with the majority of investigations, our study also had some limitations. The first limitation of our study is its retrospective nature. The second limitation is that the scores for the rules used in predicting DVT risk and APE probability and severity were seldom recorded in the available medical documentation and were calculated post-factum on the basis of the available data, which may be a source of bias. A third is that laboratory determinations were not available for all the patients analyzed; for example, blood D-dimer concentrations were available for only $72.7 \%$ of the patients studied. A fourth limitation is that follow-up was limited only to the hospitalization period and was not extended to 30 days, as in other studies. Therefore, we could not calculate the risk of 30-day mortality, as in the majority of the available papers.

\section{Conclusion}

An age-adjusted troponin cut-off and Pulmonary Embolism Severity Index (PESI) score were found to be independent risk factors of in-hospital death, but only in the whole study group and in patients without APE confirmed in CTPA; however, their clinical usefulness seems to be low due to small AUC values. The use of recent ESC Guidelines for the diagnosis and management of APE in the day-to-day real-world management of patients with suspected APE should be improved. Moreover, every patient with suspected APE needs a personalized and holistic diagnosis as well as risk-adjusted management due to DVT and APE comorbidity, as these could affect the risk of in-hospital mortality and probably decrease the prognostic power of standard APE patient stratification tools.

\section{Conflict of interest: None.}

Funding: None.

\section{References}

1. Al-Hakim R, Li N, Nonas S, et al. Evaluation and Management of Intermediate and High-Risk Pulmonary Embolism. AJR Am J Roentgenol. 2020; 214(3): 671-678, doi: 10.2214/AJR.19.21861, indexed in Pubmed: 31743047.

2. Konstantinides S, Torbicki A, Agnelli G, et al. 2014 ESC Guidelines on the diagnosis and management of acute pulmonary embolism. Kardiologia Polska. 2014; 72(11): 997-1053, doi: 10.5603/kp.2014.0211.

3. Konstantinides S, Meyer G, Becattini C, et al. 2019 ESC Guidelines for the diagnosis and management of acute pulmonary embolism developed in collaboration with the European Respiratory Society (ERS). European Respiratory Journal. 2019; 54(3): 1901647, doi: 10.1183/13993003.01647-2019.

4. Duffett L, Castellucci LA, Forgie MA. Pulmonary embolism: update on management and controversies. BMJ. 2020; 370: m2177, doi: 10.1136/bmj.m2177, indexed in Pubmed: 32759284

5. Quezada CA, Bikdeli B, Barrios D, et al. PROTECT investigators, Investigators of the PROTECT study. Assessment of coexisting deep vein thrombosis for risk stratification of acute pulmonary embolism. Thromb Res. 2018; 164: 40-44, doi: 10.1016/j.thromres.2018.02.140, indexed in Pubmed: 29476988

6. Kong WF, Wang YT, Yin LL, et al. Clinical risk stratification of acute pulmonary embolism: comparing the usefulness of CTA obstruction score and pulmonary perfusion defect score with dual-energy CT. Int J Cardiovasc Imaging. 2017; 33(12): 2039-2047, doi: 10.1007/s10554017-1188-x, indexed in Pubmed: 28612276.

7. Jen WY, Jeon YS, Kojodjojo P, et al. A New Model for Risk Stratification of Patients With Acute Pulmonary Embolism. Clin Appl Thromb Hemost. 2018; 24(9_suppl): 277S-284S, doi: 10.1177/1076029618808922, indexed in Pubmed: 30370786

8. Jia $\mathrm{D}, \mathrm{Li} \mathrm{XL}$, Zhang $\mathrm{Q}$, et al. A decision tree built with parameters obtained by computed tomographic pulmonary angiography is useful for predicting adverse outcomes in non-high-risk acute pulmonary embolism patients. Respir Res. 2019; 20(1): 187, doi: 10.1186/s12931019-1160-5, indexed in Pubmed: 31426787

9. Kozlowska M, Plywaczewska M, Koc M, et al. d-Dimer Assessment Improves the Simplified Pulmonary Embolism Severity Index for In-Hospital Risk Stratification in Acute Pulmonary Embolism. Clin Appl Thromb Hemost. 2018; 24(8): 1340-1346, doi: 10.1177/1076029618776799, indexed in Pubmed: 29806471.

10. Klingenberg R, Schlager $\mathrm{O}$, Limacher $A$, et al. Risk stratification of elderly patients with acute pulmonary embolism. Eur J Clin Invest. 2019; 49(9): e13154, doi: 10.1111/eci.13154, indexed in Pubmed: 31246275.

11. Barnes GD, Muzikansky A, Cameron S, et al. Comparison of 4 Acute Pulmonary Embolism Mortality Risk Scores in Patients Evaluated by Pulmonary Embolism Response Teams. JAMA Netw Open. 2020; 3(8): e2010779, doi: 10.1001/jamanetworkopen.2020.10779, indexed in Pubmed: 32845326.

12. Teng F, Chen $Y X, H e X H$, et al. Contribution of Quick Sequential Organ Failure Assessment Score Combined with Electrocardiography in Risk Stratification of Patients with Acute Pulmonary Embolism. Chin Med J (Engl). 2018; 131(20): 2395-2401, doi: 10.4103/03666999.243566, indexed in Pubmed: 30334523. 
13. Barco S, Mahmoudpour SH, Planquette B, et al. Prognostic value of right ventricular dysfunction or elevated cardiac biomarkers in patients with low-risk pulmonary embolism: a systematic review and meta-analysis. Eur Heart J. 2019; 40(11): 902-910, doi: 10.1093/eurheartj/ehy873, indexed in Pubmed: 30590531.

14. Elias A, Mallett S, Daoud-Elias M, et al. Prognostic models in acute pulmonary embolism: a systematic review and meta-analysis. BMJ Open. 2016; 6(4): e010324, doi: 10.1136/bmjopen-2015-010324, indexed in Pubmed: 27130162.

15. Darwish OS, Mahayni A, Patel M, et al. Cardiac Troponins in Low-Risk Pulmonary Embolism Patients: A Systematic Review and Meta-Analysis. J Hosp Med. 2018; 13(10): 706-712, doi: 10.12788/jhm.2961, indexed in Pubmed: 29694453.

16. Hobohm L, Hellenkamp K, Hasenfuß G, et al. Comparison of risk assessment strategies for not-high-risk pulmonary embolism. Eur Respir J. 2016; 47(4): 1170-1178, doi: 10.1183/13993003.01605-2015, indexed in Pubmed: 26743479.

17. Gupta R, Ammari Z, Dasa O, et al. Long-term mortality after massive, submassive, and low-risk pulmonary embolism. Vasc Med. 2020 25(2): 141-149, doi: 10.1177/1358863X19886374, indexed in Pubmed: 31845835.

18. Yamashita $Y$, Morimoto $T$, Amano $H$, et al. COMMAND VTE Registry Investigators. Usefulness of Simplified Pulmonary Embolism Severity
Index Score for Identification of Patients With Low-Risk Pulmonary Embolism and Active Cancer: From the COMMAND VTE Registry. Chest. 2020; 157(3): 636-644, doi: 10.1016/j.chest.2019.08.2206, indexed in Pubmed: 31605702

19. Ebner M, Kresoja KP, Keller K, et al. Temporal trends in management and outcome of pulmonary embolism: a single-centre experience. Clin Res Cardiol. 2020; 109(1): 67-77, doi: 10.1007/s00392-019-01489-9, indexed in Pubmed: 31065790.

20. Soriano Ld, Castro TT, Vilalva K, et al. Validation of the Pulmonary Embolism Severity Index for risk stratification after acute pulmonary embolism in a cohort of patients in Brazil. J Bras Pneumol. 2019; 45(1): e20170251, doi: 10.1590/1806-3713/e20170251, indexed in Pubmed: 30810642.

21. Vamsidhar A, Rajasekhar D, Vanajakshamma V, et al. Comparison of PESI, echocardiogram, CTPA, and NT-proBNP as risk stratification tools in patients with acute pulmonary embolism. Indian Heart J. 2017; 69(1): 68-74, doi: 10.1016/j.ihj.2016.07.010, indexed in Pubmed: 28228310.

22. Perera M, Aggarwal L, Scott IA, et al. Underuse of risk assessment and overuse of computed tomography pulmonary angiography in patients with suspected pulmonary thromboembolism. Intern Med J. 2017; 47(10): 1154-1160, doi: 10.1111/imj.13524, indexed in Pubmed: 28635149 